\title{
Distal neuroprotection system as option for right subclavian artery ostial recanalization
}

\author{
Marcin Misztal ${ }^{1}$, Piotr Pieniążek², Jakub Zasada³, Andrzej Brzychczy³, Jadwiga Nessler ${ }^{1}$, Krzysztof Żmudka $^{4}$ \\ ${ }^{1}$ Coronary Artery Disease Department, Institute of Cardiology, Jagiellonian University, John Paul II Hospital, Krakow, Poland \\ ${ }^{2}$ Department of Cardiac and Vascular Diseases, Institute of Cardiology, Jagiellonian University, John Paul II Hospital, Krakow, Poland \\ ${ }^{3}$ Vascular Surgery Department, John Paul II Hospital, Krakow, Poland \\ ${ }^{4}$ Department of Haemodynamics and Angiocardiography, Institute of Cardiology, Jagiellonian University, John Paul II Hospital, Krakow, Poland
}

Postep Kardiol Inter 2013; 9, 4 (34): 341-343

DOI: $10.5114 / p w k i .2013 .38862$

\begin{abstract}
A bstract
We present the case of a 49-year-old woman with neurological symptoms and severe atherosclerosis of aortic arch branches affecting subclavian and carotid arteries. Our patient has a history of transient ischemic attack and recurrent paresthesias of her right arm. We present a double access intervention using a distal embolic neuroprotection device during ostial right subclavian artery recanalization. We recommend that use of an embolic protection device in right subclavian artery ostial recanalization should be considered.
\end{abstract}

Key words: subclavian artery, transient ischemic attac, distal neuroprotection system.

\section{Introduction}

Occlusive disease of the arch vessels can be manifested within different neurological symptoms. Atherosclerosis of aortic arch branches is very common among patients suffering from coronary artery disease (4-7\%) and peripheral arterial disease (PAD; 11.5\%) [1, 2]. In a large cohort study done by Shadman et al. subclavian stenosis correlation with smoking history, high systolic blood pressure, HDL levels and PAD was confirmed [3]. An interesting study by Kablak-Ziembicka et al. confirmed the correlation between severity of coronary artery disease (CAD) and aortic arch branches stenosis. The authors found that none of the patients with normal coronary arteries had severe stenosis of the extracranial arteries and $16.6 \%$ of patients with three vessel CAD presented severe stenosis of aortic arch arteries [4].

Innominate or subclavian artery occlusion is one of the most challenging lesions qualified for invasive treatment. An endovascular approach to these lesions is now preferred in stenoses and occlusions of the brachiocephalic vessels. The question of using a cerebral protection device in subclavian and innominate interventions is still open [5]. Interventions in the innominate and right subclavian artery expose patients to the risk of embolization within carotid and vertebral circulation [1]. There are a few options for reducing the risk of embolization, i.e. proper oral or intravenous pharmacotherapy and embolic protection devices. In most interventions performed with the femoral access the use of a protection device seems to be unnecessary. The use of a cerebral protection device in the right subclavian and innominate artery performed through the brachial approach is less established [6].

The case of a 49-year-old woman with neurological symptoms and atherosclerosis of aortic arch branches affecting subclavian and carotid arteries will be presented. The patient underwent a double access intervention using a distal embolic protection device during ostial right subclavian artery (RSA) recanalization.

\section{Case report}

A 49-year-old woman was referred to our department in February 2012 after transient ischemic attack (TIA) of the left hemisphere observed in January 2012. Prior to TIA the patient reported transient paresthesias of the right upper limb and left part of her face and very acute and short speech problems. Doppler ultrasound performed in

\section{Corresponding author:}

Marcin Misztal MD, PhD, Coronary Artery Disease Department, Institute of Cardiology, Jagiellonian University, John Paul II Hospital, 80 Prądnicka St, 31-202 Krakow, Poland, tel.: +48 606265 579, fax: +48 1263367 44, e-mail: marcin.misztal1@gmail.com

Received: 9.09.2013, accepted: 10.10.2013. 


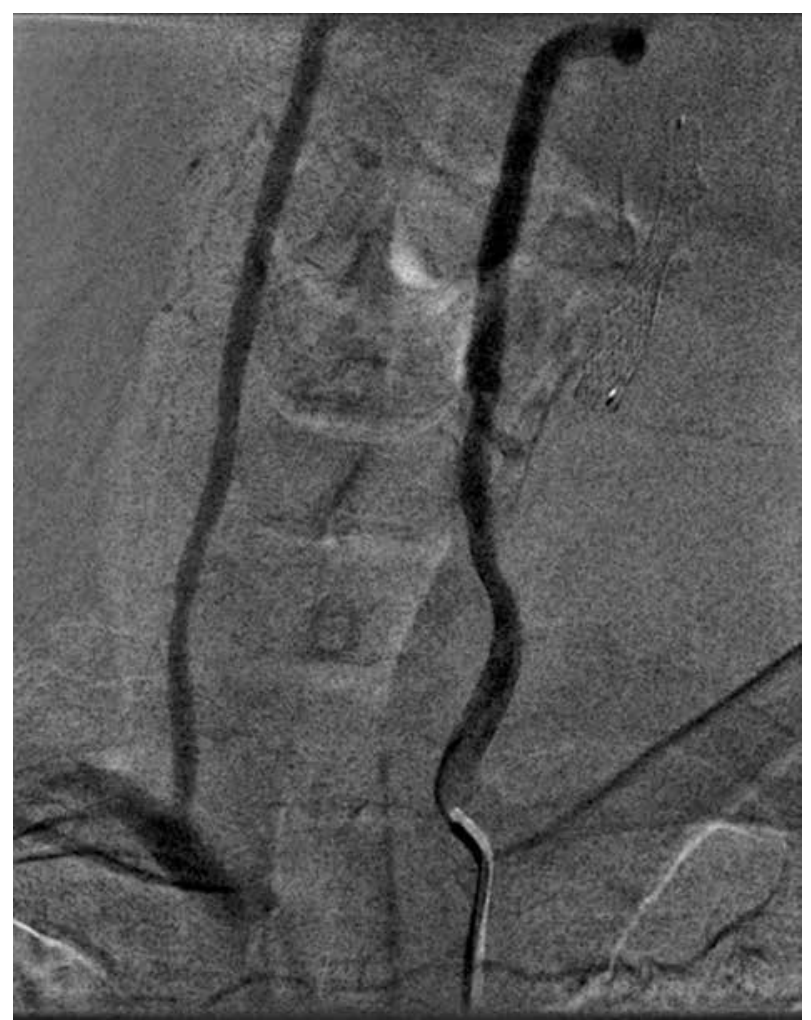

Fig. 1. Right subclavian steal syndrome (SSS) visualized from left vertebral angiography

the department of neurology revealed critical stenosis $>90 \%$ (PSV - $7.5 \mathrm{~m} / \mathrm{s}$ ) of the left internal carotid artery (LICA) and occlusion of the right subclavian artery (RSA) with subclavian steal syndrome (SSS). In the brain computed tomography (CT) there were no signs of ischemic lesions. Blood pressure measurements confirmed $40-50 \mathrm{~mm} \mathrm{Hg}$ asymmetry in the systolic blood pressure between arms. The patient presented a few atherosclerosis risk factors, e.g. hypertension, hyperlipidemia, obesity, diabetes melli-

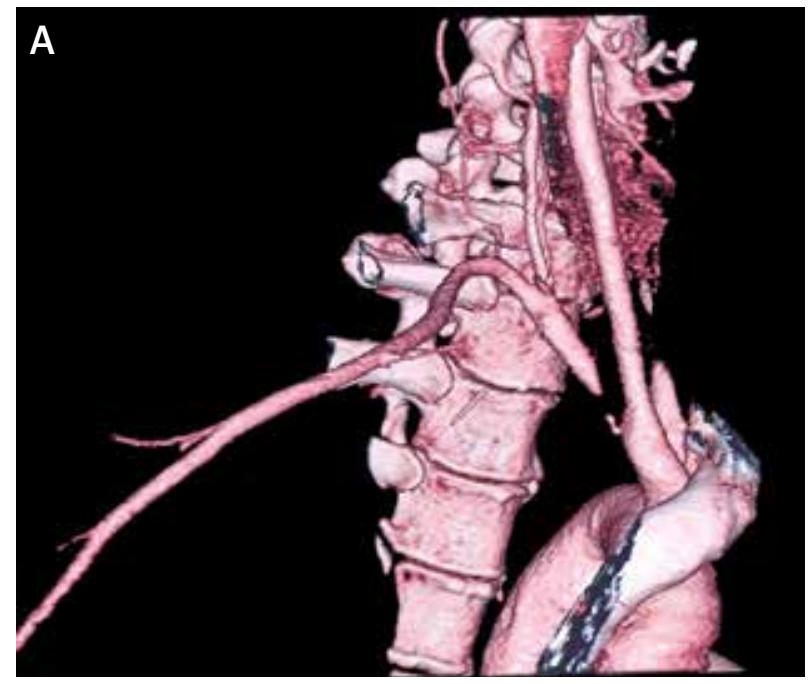

Fig. 2. A - MPR CT reconstruction of innominate, right subclavian and common carotid arteries. B - CT scan (MIP) showing ostial occlusion of right subclavian artery (no signs of calcifications) tus and nicotine abuse. As a first step we decided to perform carotid artery stenting (CAS) as a primary prevention of the stroke. The procedure was done at the beginning of March 2012 using a proximal protection Mo.Ma 8 Fr device (Medtronic, US) and implantation of a self-expanding Cristallo Ideale 6-9/40 mm tapered stent (Medtronic, US). During catheterization a steal syndrome of the right subclavian artery through the left vertebral artery was visualized (Figure 1). After carotid artery stenting (CAS-LICA) the patient was put on dual antiplatelet therapy with a high dose of statin and was discharged home with scheduled angio-CT of innominate and right subclavian arteries. Three months later the patient visited the out-patient clinic complaining of right arm weakness and claudication, loss of precision within the right hand and recurrent dizziness. She also presented her CT scans confirming short, soft occlusion of the orifice of the RSA (Figure 2).

Due to the patient's complaints, high interarm systolic blood pressure (SBP) difference and young age we qualified her for a recanalization of the subclavian artery. After neurological assessment in September 2012 the intervention was performed. We decided to use two different access sites: first the surgical right brachial artery was opened and then the endovascular right common femoral artery site. After insertion of a 6 Fr Judkins Right (Cordis, US) guiding catheter through brachial access the occlusion was crossed with a Cross-IT 400XT wire (Abbott Laboratories, Illinois, US) and Maverick 2.0/20 mm (Boston Scientific, Massachusetts, US) balloon catheter (Figure 3 A). Our next step was to introduce the distal protection system Filter Wire EZ (Boston Scientific, Massachusetts, US) to the right common carotid artery to protect cerebral arteries from embolization (Figure $3 \mathrm{~B}$ ). There was a risk of brain embolization due to the plaque location and composition. Next, one predilatation with a balloon catheter was performed and then a chromium-cobalt Omnilink-

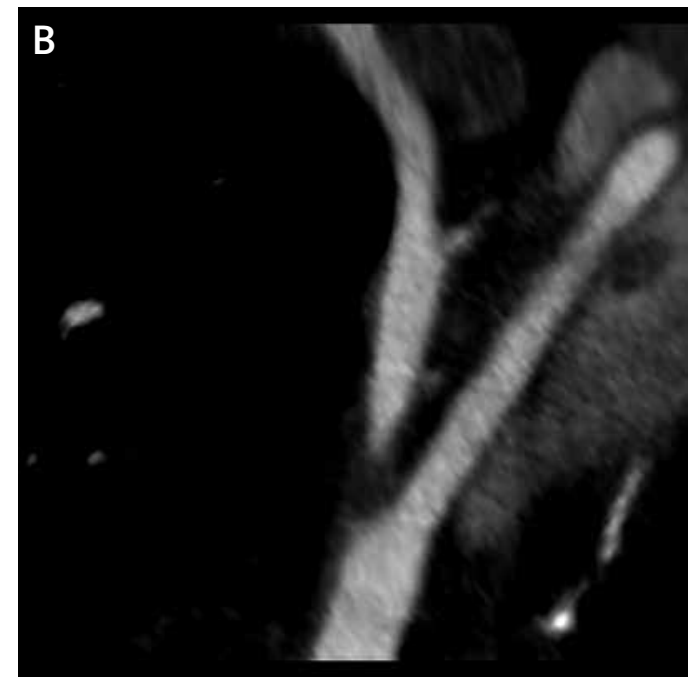



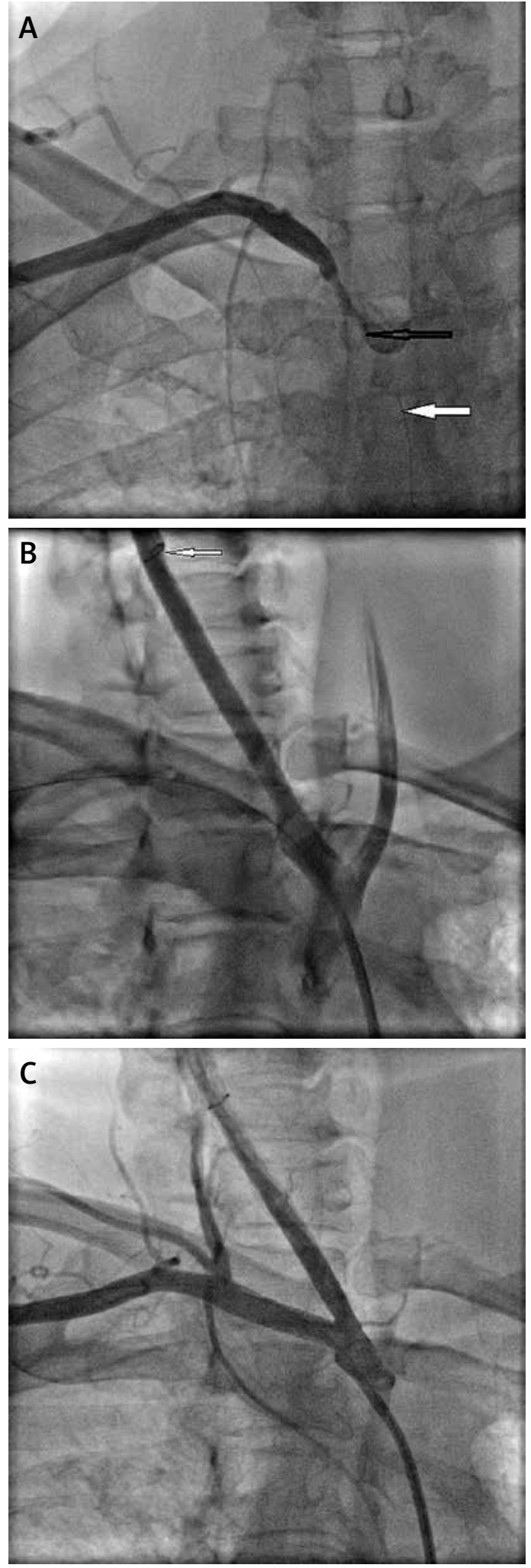

Fig. 3. A - Crossing the occlusion with Maverick 2.0/20 mm balloon catheter (black arrow) and Cross-IT 400XT guide wire (white arrow). B - Preparing for stenting with Filter WireEZ in place (white arrow). C - Final angiographic result after stenting (filter still in place)
Elite 6.0/19 mm stent was implanted (Abbott Laboratories, Illinois, US). Finally we postdilated the proximal part of the stent with $16 \mathrm{~atm}$ pressure over $45 \mathrm{~s}$ and decided to finish the procedure with an optimal angiographic result (Figure $3 \mathrm{C}$ ). When retrieving the filter we did not find any visible embolic debris. The procedure was uneventful and both access sites were properly closed (the brachial site surgically and the femoral site with an AngioSeal $8 \mathrm{Fr}$ (St. Jude Medical, US) closure device). The patient presented no complaints during the intervention and no neurological symptoms after the procedure. The day after we confirmed our result clinically. The ultrasound test was performed and the patient was discharged home. Six months later on follow-up the patient did not present any signs of right arm ischemia, and interarm blood pressure difference was less than $10 \mathrm{~mm} \mathrm{Hg}$.

\section{Discussion}

Percutaneous transluminal angioplasty (PTA) since its first presentation in 1980 is still encouraging and has become a strong alternative to surgical revascularization. It should be emphasized that endovascular techniques are the first choice treatment in aortic arch vessel disease.

Embolic protection devices are routinely used during stenting of the extracranial carotid arteries. Indications for the use of a cerebral protection device in subclavian and innominate interventions are still unclear. There are still very few reports on distal protection of the vertebral and carotid arteries during innominate artery recanalization $[5,6]$.

As we present in our work, we think that the use of an embolic protection device in right subclavian artery ostial recanalization should be considered. Special indications for using a distal protection system are: antegrade access, soft/fibrotic plaque and ischemic lesions found in CT or MRI of the brain.

\section{References}

1. Cronenwett JL, Johnston KW. Rutherford's Vascular Surgery. $7^{\text {th }}$ ed. Saunders Elsevier, Philadelphia 2010: 1545-1556.

2. English JA, Carell ES, Guidera SA, Tripp HF. Angiographic prevalence and clinical predictors of left subclavian stenosis in patients undergoing diagnostic cardiac catheterization. Catheter Cardiovasc Interv 2001; 54: 8-11.

3. Shadman R, Criqui MH, Bundens WP, et al. Subclavian artery stenosis: prevalence, risk factors and association with cardiovascular disease. J Am Coll Cardiol 2004; 44: 618-623.

4. Kablak-Ziembicka A, Tracz W, Przewlocki T, et al. Association of increased carotid intima-media thickness with the extent of coronary artery disease. Heart 2004; 90: 1286-1290.

5. Przewlocki T, Kablak-Ziembicka A, Pieniazek P, et al. Determinants of immediate and long-term results of subclavian and innominate artery angioplasty. Catheter Cardiovasc Interv 2006; 67: 519-526.

6. Stiefel MF, Park MS, McDougall CG, Albuquerque FC. Endovascular treatment of innominate artery occlusion with simultaneous vertebral and carotid artery distal protection: case report. Neurosurgery 2010; 66: E843-E844. 